\title{
Comprehensive Analysis of Hydrogen, Deuterium, Tritium and Isotopic Ratios of Other Light Elements in Neutron Irradiated TPBAR Components
}

\author{
Arun Devaraj $^{1 *}$, Bethany Matthews ${ }^{2}$, Bruce Arey $^{2}$, Elizabeth $\mathrm{Kautz}^{3}$, Gary Sevigny ${ }^{2}$ and David Senor ${ }^{2}$ \\ 1. Physical and Computational Sciences Directorate, Pacific Northwest National Laboratory, Richland, \\ WA, USA. \\ 2. Energy and Environment Directorate, Pacific Northwest National Laboratory, Richland, WA, USA. \\ 3. National Security Directorate, Pacific Northwest National Laboratory, Richland, WA, USA. \\ *Corresponding author: arun.devaraj@pnnl.gov
}

Tritium, a hydrogen isotope of significant importance, is generated by neutron irradiation of Tritium Producing Burnable Absorber Rods (TPBARs), which are specifically designed to produce and capture tritium [1, 2]. A schematic of TPBAR is given in figure 1 (a). Inside each TPBAR there are lithium aluminate pellets, enriched in ${ }^{6} \mathrm{Li}$ that produces tritium upon neutron irradiation. Tritium is then absorbed by a Zircaloy-4 getter tube that surrounds the $\mathrm{LiAlO}_{2}$ pellet. These components are encapsulated inside a stainless steel cladding which is expected to prevent the diffusion of tritium towards outside. Understanding how various elements in each component gets redistributed under neutron irradiation is a challenging task since it involves quantitatively analyzing changes in distribution of light elements like $\mathrm{Li}, \mathrm{H}$ isotopes, $\mathrm{He}, \mathrm{O}$ and other heavy elements. Most traditional characterization methods including electron microscopy and diffraction studies using X-rays do not have sufficient sensitivity or spatial resolution needed for this study, especially when it comes to light elements. Hence in this work we utilized Atom probe tomography to systematically analyze the solute distribution in both as-fabricated and irradiated TPBAR components to obtain a comprehensive understanding of the microstructural evolution under irradiation.

The TPBAR components both before and after irradiation were sectioned and prepared for FIB lift-out using various procedures, to minimize the contamination resulting from the radioactivity of these materials as well as to ensure that the materials are not modified by the sample preparation procedures. Then using a Helios FIB based lift-out and annular milling, APT needle specimens were prepared in PNNL radiochemical processing laboratory. The APT data was collected using CAMECA LEAP 4000 XHR Atom probe tomography system.

The ion map of $\mathrm{LiAlO}_{2}$, Zircaloy-4 and 316 stainless steel cladding in the as-fabricated and after irradiation state are provided in figure 1(b). Detailed mass-to-charge spectra analysis of $\mathrm{LiAlO}_{2}$ pellets after irradiation revealed clear presence of tritium in the form of a distinct peak at 3Da after irradiation as shown in figure 1(c). Additionally the analysis of ${ }^{6} \mathrm{Li}$ and ${ }^{7} \mathrm{Li}$ isotopic ratio revealed the ability of APT in measuring the ${ }^{6} \mathrm{Li}$ enrichment quantitatively both before irradiation and how it changes after irradiation. The APT analysis of Zircaloy-4 before irradiation, after $\mathrm{H}$ loading, after Deuterium loading and after neutron irradiation highlighted distinct difference in mass-to-charge ratio spectra. The comparison of APT results from 316 stainless steel cladding before and after irradiation revealed significant irradiation induced precipitation and presence of trace amount of tritium. These results provided comprehensive understanding of how individual element distribution in each TPBAR component changes as a result of irradiation while additionally revealing the spatial distribution of hydrogen isotopes. 
There are however challenges when it comes to quantifying the concentration of hydrogen isotopes in these TPBAR component materials due to the persistent background $\mathrm{H}$ peaks at 1 and $2 \mathrm{Da}$ even in the materials without any $\mathrm{H}$ isotopes. Hence a global discussion of both strengths and challenges in analyzing $\mathrm{H}$ isotopes and light elements in materials using atom probe tomography is crucial for potentially expanding the application of APT to a variety of technical areas ranging from national security, hydrogen storage, nuclear materials and hydrogen embrittlement of structural alloys in variety of applications.

\section{References:}

[1] KA Burns, EF Love and CK Thornhill, Description of the Tritium- Producing Burnable Absorber Rod for the Commercial Light Water Reactor TTQP-1-015, Revision 19, PNNL report, PNNL-22086, (2012).

[2] DJ Senor, Science and Technology in Support of the Tritium Sustainment Program, PNNL report, PNNL-27216, (2018).
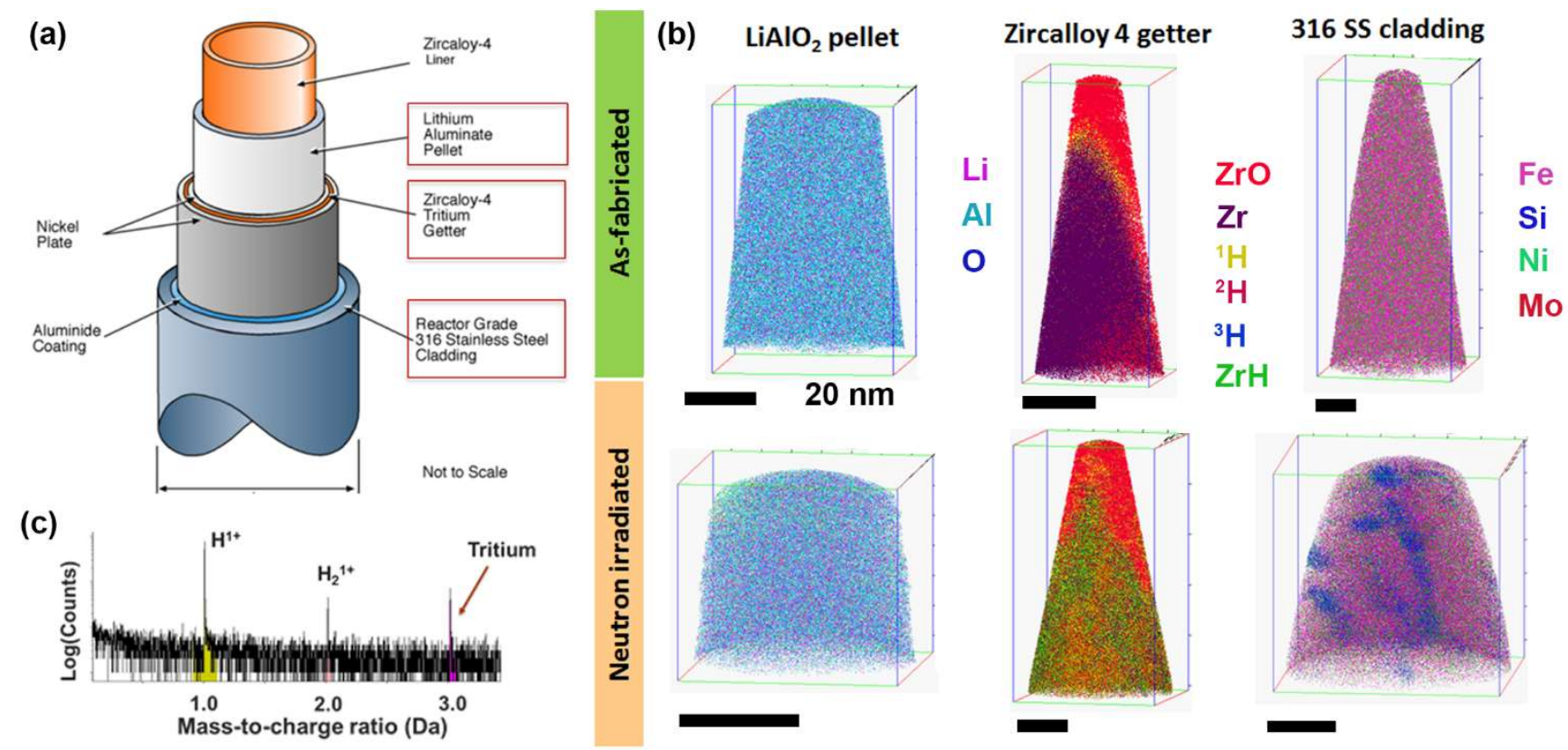

Figure 1. (a) Schematics of TPBAR components (b) the comparison of ion maps of $\mathrm{LiAlO}_{2}$, Zircaloy-4 getter and 316 stainless steel cladding in the as-fabricated state and after neutron irradiation. The scale bars corresponds to $20 \mathrm{~nm}$. The mass-to-charge spectra of irradiated LiAlO2 pellet in the 0-4 Da region showing distinct peak at 3 Da corresponding to Tritium. 